\title{
Coproduction et partage de documentation francophone de qualité sur le prototypage rapide : une activité d'inspiration socioconstructiviste soutenue par les TIC
}

\section{Sylvie Doré}

École de technologie supérieure

sylvie.dore@etsmtl.ca

\section{Compte rendu de pratique}

\section{Résumé}

Le cours « MEC626 - Prototypage rapide » est centré sur une activité d'apprentissage nommée « séminaire " grâce à laquelle des étudiants coproduisent de la documentation francophone de qualité formant une partie importante des notes de cours. D'inspiration socioconstructiviste, cette activité apprend aux étudiants à utiliser des outils et à développer des savoir-faire qui leur seront utiles lors de l'exercice de leur profession. Au total, sept technologies sont mises en œuvre pour soutenir l'activité. Parmi celles-ci, mentionnons une variété de moteurs de recherche utilisés pour trouver l'information pertinente, un logiciel de gestion de notices bibliographiques servant à classifier l'information et à produire une base de données collective et le transfert FTP permettant l'échange de documents et agissant comme vitrine de travaux. Le séminaire permet non seulement aux étudiants de se familiariser avec le contenu du domaine, mais il leur offre également l'occasion de maîtriser un ensemble d'outils et de méthodes exploitant les TIC, et de vivre une expérience à la fois significative et pertinente.

\section{Abstract}

The course MEC626 Prototypage rapide is centred around a learning activity called the Seminar, in which students coproduce high-quality French-language documentation that makes up a large part of the course notes. Social-constructivist in inspiration, this activity mobilizes the students to use tools and develop knowhow that will be useful to them in the practice of their profession. In all, seven technologies support the activity. Most noteworthy among them are: a variety of search engines that are used to find relevant information; a bibliographical management software that is useful for categorizing information and producing a collective database; and FTP transfer which allows the exchange of documents and serves as a showcase for student assignments. Not only does the Seminar enable students to familiarize themselves with the subject matter, it also offers them the opportunity to master a set of ICTbased tools and methods, while providing them with an experience that is both significant and relevant.

\section{Introduction}

À l'été 2002, l'auteure a procédé à une refonte ${ }^{1}$ importante du cours « MEC626 - Prototypage rapide" (consultez l'annexe 1 pour une définition de cette technologie). Il s'agit d'un cours optionnel de trois crédits inscrit au programme de génie mécanique de l'École de technologie supérieure (ÉTS). Il s'offre sur campus en présence.

Cet article rend compte d'une activité d'inspiration socioconstructiviste menant à la coproduction, par les étudiants, de documentation francophone de qualité sur le prototypage rapide où les TIC jouent un rôle facilitateur dans la recherche, l'organisation, le traitement et la diffusion de l'information. Après avoir exposé les principaux objectifs ayant guidé la conception de cette activité, nommée "séminaire", une description de son déroulement et des modalités d'évaluation est présentée. Les points saillants de l'évaluation de l'activité précèdent un retour sur l'aspect socioconstructiviste et la conclusion. 


\section{Motifs du changement}

Plusieurs facteurs ont motivé la refonte du cours. Trois d'entre eux, présentés sous forme d'objectifs, sont détaillés ici. Alors que le premier s'applique à l'ensemble des activités du cours, les deux derniers sont plus spécifiques à l'activité de séminaire.

1. Introduire des activités d'apprentissage d'inspiration socioconstructiviste. Selon Lafortune et Deaudelin (2002), le socioconstructivisme se décline en trois volets : constructiviste, social et interactif. Contrairement au behaviorisme où la personne apprenante répond à un stimulus de l'environnement (p. ex., des consignes à réaliser, un exposé magistral, la réalisation d'exercices), le constructivisme l'encourage à s'engager dans une recherche de sens (Perkins, 1991), à construire son savoir. Ainsi, elle est responsable de ses apprentissages. Du coup, ceci implique une redéfinition du rôle de la personne enseignante. Cette dernière n'est plus une experte qui transmet son savoir, mais elle devient un guide, un coach qui remet en question les constructions existantes de la personne apprenante. Les constructivistes considèrent qu'il n'y a pas de réalité objective, que la connaissance est un construit qui fait consensus à un moment donné de l'histoire et qui est appelé à évoluer. L'aspect social et les interactions entre les individus lors desquelles chacun confronte sa représentation d'une connaissance à celle des autres sont donc au cour du constructivisme. Autrement dit, selon Sauvé (2000) qui cite Marie-France Legendre: « La socialisation contribue à donner un sens à l'apprentissage». Mais il ne s'agit pas seulement d'interagir avec les autres. Le volet interactif s'étend au milieu dans lequel l'individu évo- lue. Or pour les ingénieurs, ce milieu est éminemment supporté par les TIC, d'où l'importance d'inclure ces technologies dans les activités d'apprentissage.

2. Renforcer l'acquisition d'habiletés transversales. Les compétences communicationnelles et informationnelles sont spécifiquement visées par l'activité de séminaire. Le Conseil canadien des ingénieurs (2003), dans son dernier rapport sur la diplomation, mentionne bien «que pour une progression de carrière en génie, il est essentiel d'acquérir des compétences dans un domaine particulier du génie de même que de posséder de solides habiletés en communication, en planification et en qualité de chef " (p. 2). Bien que les étudiants de l'ÉTS bénéficient d'un cours de communication lors de leur première année d'études, leur compétence en cette matière est rarement réactivée de façon formelle ou réévaluée dans les cours de spécialisation. Il s'ensuit que peu d'étudiants progressent pour atteindre un niveau de maîtrise de cette compétence. En ce qui concerne la compétence informationnelle, un changement important a été noté depuis quelques années. Il y a à peine cinq ans, il fallait former les étudiants à l'utilisation de moteurs de recherche sur Internet. Aujourd'hui, les étudiants ont de la difficulté à effectuer une recherche bibliographique de qualité car ils se limitent presque exclusivement à l'information glanée sur le Web. La Conférence des recteurs et des principaux des universités du Québec (CREPUQ) a d'ailleurs récemment publié une étude (Mittermeyer et Quirion, 2003) portant sur le degré de maitrise des connaissances de base requises pour effectuer un travail de recherche documentaire chez les étudiants de premier cycle. Cette étude a constaté que "bon nombre d'étudiants semblent mal connaître ou ne pas connaître du tout les éléments de base du processus de recherche documentaire " (p.7), les principales conséquences étant :

- "La difficulté à repérer l'information pertinente : peu ou pas de documents ou un trop grand nombre de documents sont repérés. L'utilisation de concepts inappropriés, la méconnaissance de la structure et du contenu du catalogue ainsi que du vocabulaire contrôlé, une stratégie de recherche déficiente et l'incapacité à interpréter une référence bibliographique sont autant d'éléments qui nuisent au repérage des documents.

- L'inefficacité : diverses pistes sont explorées sans résultat satisfaisant. Le procédé par essais et erreurs enlève du temps à l'étudiant pour la lecture des documents, l'appropriation de leur contenu et la rédaction de ses travaux.

- Potentialité de plagiat par ignorance des règles d'ethique documentaire, en particulier celles concernant les références des documents cités et consultés.»(p.7)

3. Produire de la documentation francophone de qualité. Il existe très peu de matériel de référence francophone dans le domaine du prototypage rapide. De plus, les principales sources d'information disponibles en anglais sont très coûteuses. Les informations deviennent rapidement désuètes tant le prototypage rapide connaît une évolution fulgurante. La rédaction de matériel pédagogique devient alors une tâche extrêmement énergivore et d'une utilité limitée dans le temps pour les étudiants. 
En invitant les étudiants à coproduire de la documentation à jour sur le prototypage rapide, et ainsi constituer une partie importante des notes de cours, et à en diffuser le contenu, les deux derniers objectifs sont visés. La qualité de la documentation, sans être garantie, est améliorée en introduisant une boucle de rétroaction sous forme de critique constructive entre la rédaction de deux versions des documents. Une description détaillée de l'activité et du mode d'évaluation des apprentissages permettra de mieux en saisir la nature socioconstructiviste. Mentionnons pour l'instant que presque tous les cours magistraux ont été remplacés soit par des discussions de groupe, soit par la réalisation des activités. Un cahier de l'apprenant a été rédigé de façon à refléter cette structure et contient des consignes suffisamment détaillées pour que les activités puissent être réalisées de façon autonome. Un site Web complète le matériel pédagogique.

\section{Description du déroulement du séminaire}

Le séminaire, au cours duquel des équipes de deux à trois étudiants sont invitées à faire une recherche bibliographique sur une famille de procédés de prototypage ou d'outillage rapide, forme le cour du cours. Chaque équipe diffuse les résultats de cette recherche sous forme de rapport et lors d'une séance d'affiche à laquelle toute la communauté de l'ÉTS est invitée. Le scénario pédagogique est décrit ci-dessous.

Étape 1: Rédiger une première version $d u$ rapport. Chaque équipe se voit attribuer une famille de procédés de prototypage ou d'outillage rapide. Afin d'éviter que seuls les sites Internet ne soient exploités comme source d'information, une formation de quatre heures, répartie sur deux semaines, est offerte en laboratoire informatique par une bibliothécaire, spécialiste en recherche documentaire. La formation porte sur les différentes sources bibliographiques (sites Web, monographies, articles scientifiques sur supports papier et électronique, bases de données bibliographiques, incluant les répertoires de thèses et de brevets), sur la valeur scientifique de ces sources, sur les outils de recherche électronique et sur un outil de gestion des notices bibliographiques (EndNote). Les étudiants effectuent une recherche, assimilent et organisent l'information et produisent un premier rapport avec le logiciel Word. Le logiciel EndNote est utilisé non seulement pour organiser l'information, mais aussi pour générer automatiquement la bibliographie du rapport.

Étape 2: Critiquer un rapport. Cette étape débute avec une formation sur la critique constructive. Les étudiants complètent un formulaire sur leurs attitudes face à la critique. S'ensuit une discussion où chacun est invité à partager ces attitudes. Il en ressort deux constats : 1) Les étudiants accordent peu de confiance à la capacité de leurs pairs de critiquer adéquatement leur travail, seul l'avis d'un supérieur, en l'occurrence la personne enseignante, étant considéré comme légitime; et 2) Le seuil de tolérance à la critique est très variable d'un individu à l'autre, d'où l'importance que l'on doit accorder à la forme de la critique. Un court exposé sur la forme et le contenu d'une critique constructive clôt cette formation.

Chaque étudiant est ensuite invité à critiquer le rapport rédigé par une autre équipe. En plus de produire une synthèse de leur critique, ils doivent utiliser les outils de révision et d'insertion de commentaires du logiciel Word pour formuler des remarques précises directement dans le rapport. Notons qu'aucun étudiant n'avait jamais utilisé ces fonctionnalités auparavant.

Une question se pose alors : sous quelle forme échanger le rapport, puis sa criti- que? Trois solutions, exploitant les TIC, sont envisageables:

- Les étudiants échangent les documents par courriel;

- Chaque équipe transmet son rapport à l'enseignant, qui le dépose sur le site Web du cours où il sera ensuite récupéré;

- Chaque équipe dépose son rapport sur un site FTP (File Transfer Protocol).

Parce que l'auteure désirait créer une vitrine des travaux où chacun pourrait récupérer les travaux des autres, tout en se déchargeant de la tâche fastidieuse de déposer elle-même les travaux sur le site, la troisième solution fut retenue. Ainsi, chaque étudiant récupère le rapport qui lui a été assigné, le critique et dépose la version critiquée sur le site FTP.

Étape 3: Rédiger une deuxième version $d u$ rapport. Chaque équipe récupère les critiques de son rapport original. Puisque la critique est une tâche réalisée individuellement, chaque équipe bénéficie des commentaires de la professeure et de ceux de deux ou trois étudiants. Une deuxième version du rapport est produite et transmise électroniquement par courriel à la professeure avec la librairie EndNote contenant les notices bibliographiques utilisées pour préparer le rapport. Mentionnons une énorme augmentation de la qualité des rapports entre les deux versions, soulignant la pertinence de la critique constructive. À l'aide de l'outil " commentaire» de Word, la professeure relève les erreurs de contenu qui auraient pu subsister dans le rapport. Ces versions corrigées sont colligées en "actes du séminaire», qui forment une partie des notes de cours. Les actes sont ensuite distribués à chaque étudiant sous forme papier. La forme électronique est également déposée sur le site FTP. Les librairies EndNote de chaque équipe sont concaténées afin de former une 
seule librairie qui est également mise à la disposition de tous les étudiants.

Ce scénario exige un échange de plusieurs documents. Afin d'éviter la confusion, il est de mise de mettre en place les trois mesures suivantes :

- Développer une nomenclature pour les noms de fichier et la transmettre aux étudiants;

- Préparer une liste des noms des documents que chaque étudiant devra déposer et récupérer;

- Créer des sous-répertoires de dépôt pour chaque type de document créé: un pour le dépôt de la première version du rapport, un pour la critique, un pour la deuxième version du rapport et le dernier pour la deuxième version corrigée.

Étape 4: Participer au séminaire. Pendant que la professeure corrige la deuxième version des rapports, chaque équipe prépare une affiche. La séance d'affiche poursuit deux buts : faire expérimenter aux étudiants une nouvelle forme de communication et diffuser l'information contenue dans les rapports le plus rapidement possible. Ce mode de communication est intéressant car il allie à la fois le développement de la communication orale et rédactionnelle tout en laissant une place à la créativité. Bien que l'affiche puisse prendre différentes formes, les étudiants sont encouragés à utiliser un logiciel de mise en forme permettant l'impression numérique. Les logiciels PowerPoint, Visio et Photoshop peuvent être utilisés à cette fin.

Étape 5: Assimiler l'information. Lors de la réalisation des trois premières étapes, les étudiants sont exposés à deux familles de procédés : celui qui leur a été assigné et celui du rapport qu'ils auront critiqué. À l'étape 4, ils auront rapidement pris connaissance des autres procédés qu'ils devront maintenant assimiler. Afin de les aider dans cette tâche, des gabarits électroniques pour la réalisation de fiches-résumés sont mis à leur disposition sur le site Web. De plus, des activités de classification sont réalisées en classe.

Le Tableau 1 résume les technologies de l'information utilisées pour soutenir la réalisation du scénario pédagogique de l'activité de séminaire. On y recense sept technologies différentes. À travers l'activité, non seulement les étudiants améliorent-ils leurs méthodes de gestion de l'information et développent-ils leur esprit critique et leurs habiletés de communication de l'information, mais ils découvrent également de nouveaux outils.

\section{TECHNOLOGIES UTILISÉES}

TÂCHES

Technologies Web

\begin{tabular}{|c|c|}
\hline $\begin{array}{l}\text { Moteurs de recherche de sites Web, de } \\
\text { bases de données bibliographiques (p.ex., } \\
\text { Compendex), de revues savantes (p.ex., } \\
\text { Emerald Fulltext, Wiley InterScience, etc.), } \\
\text { de banques de brevets (Office de la propriété } \\
\text { intellectuelle du Canada), de thèses (Digital } \\
\text { Dissertations), etc. }\end{array}$ & Recherche d'information \\
\hline Transfer FTP (WinFTP) & Échange de fichiers, vitrine \\
\hline Courriel & Envoi de fichiers à la professeure \\
\hline Téléchargement & $\begin{array}{l}\text { Récupération de matériel pédagogique (gabarits, } \\
\text { complément de consignes, etc.) }\end{array}$ \\
\hline \multicolumn{2}{|l|}{ Logiciels } \\
\hline $\begin{array}{l}\text { Logiciel de gestion de notices bibliographiques } \\
\text { (EndNote) }\end{array}$ & $\begin{array}{l}\text { Création d'une bibliographie, citation des sources } \\
\text { bibliographiques }\end{array}$ \\
\hline $\begin{array}{l}\text { Fonctions « commentaire» } \\
\text { et « révision» de Word }\end{array}$ & Critique \\
\hline $\begin{array}{l}\text { Logiciel de mise en page graphique } \\
\text { (PowerPoint, Visio, Photoshop, etc.) }\end{array}$ & Production d'une affiche \\
\hline
\end{tabular}

Tableau 1. Ensemble des technologies de l'information déployées pour soutenir la réalisation du séminaire

\section{Évaluation des apprentissages}

Trente pour cent (30\%) de la note globale du cours, décomposée comme suit, est accordée au séminaire:

- $10 \%$ pour la première version du rapport;

- $5 \%$ pour la qualité de la critique du rapport d'un collègue;

- $10 \%$ pour la deuxième version du rapport;

- $5 \%$ pour la réalisation de l'affiche.

La maîtrise des TIC est vérifiée de façon indirecte en spécifiant des critères d'évaluation nécessitant leur utilisation. Par exemple, une partie de l'évaluation des rapports porte sur la qualité de la bibliographie. Les sources doivent être diversifiées et de qualité. L'utilisation de moteurs de recherche dans différentes banques et l'élaboration de stratégies efficaces de- 
viennent donc essentielles dans de telles conditions. De plus, à la fin de la session, chaque équipe doit remettre sa librairie EndNote contenant l'ensemble des références utilisées pour rédiger son rapport.

Avant de pouvoir procéder à la critique, les étudiants doivent nécessairement déposer leur premier rapport sur le site FTP puis récupérer le rapport qui leur a été assigné pour la critique. Ils doivent donc tous maîtriser l'outil. De plus, la critique est évaluée, en partie, sur l'utilisation pertinente des fonctions de Word tout au long du texte.

\section{Évaluation de l'activité}

Le cours a été soumis à deux évaluations formatives et à une évaluation sommative. L'évaluation sommative est réalisée par questionnaire standardisé et est organisée par l'administration de l'École. Les résultats de cette évaluation n'apportant aucune nouvelle information, seuls les résultats des deux évaluations formatives sont présentés.

La première évaluation formative a eu lieu à la quatrième semaine. Sur une feuille de papier, les étudiants décrivent trois points qu'ils considèrent comme positifs et trois points qu'ils désirent voir améliorés. Il ressort de cette première évaluation que la plupart des étudiants apprécient une approche pédagogique active, fondée sur des activités d'apprentissage, mais qu'ils ont de la difficulté à identifier les tâches à effectuer chaque semaine. Cette situation a été réglée en leur expliquant comment utiliser les outils de gestion (plan de cours, calendrier des activités, diagramme de gestion de projet et consignes détaillées des activités) mis à leur disposition. De plus, un rappel des tâches à effectuer et des dates de tombée des productions attendues est affiché hebdomadairement sur le site Web du cours.
La deuxième évaluation formative a eu lieu dans les minutes qui ont suivi l'examen final. Douze étudiants sur quinze y ont répondu. Le but principal de cette évaluation était de vérifier si les étudiants jugeaient les connaissances et activités pertinentes. En effet, selon Glasersfeld (1994), pour qu'il $y$ ait construction de connaissance, les apprenants doivent être placés dans une situation d'apprentissage significative (dans le cas qui nous intéresse, produire de la documentation francophone de qualité sur le prototypage rapide) et pertinente à l'égard des pratiques socialement établies. Dans un premier temps, les apprenants ont été invités à classer, par ordre décroissant, l'importance que revêtent pour eux les connaissances acquises dans le cours pour leur future vie professionnelle. Dans un deuxième temps, ils se sont prononcés sur la pertinence de ces connaissances. Une section libre complète le questionnaire. Bien que peu d'étudiants y aient participé et que le questionnaire comporte des limites, quelques constats intéressants ressortent de cette évaluation:

- Neuf étudiants jugent les connaissances acquises sur le prototypage rapide plus importantes que la méthode nécessaire à la mise à jour de ces connaissances (recherche et gestion d'information). L'auteure croyait que le seul fait de vivre l'expérience du séminaire aurait été suffisant pour que les étudiants prennent conscience que, dans un contexte où les connaissances deviennent rapidement périmées, il est plus important de maîtriser les outils et la méthodologie leur permettant d'appliquer un processus de construction de connaissances plutôt que d'acquérir ces connaissances. Cette hypothèse s'est révélée fausse. L'activité mériterait d'être raffinée, possiblement grâce à des discussions sur le sujet, afin de renverser la perception des étudiants sur ce point.

- Même les connaissances jugées moins importantes pour leur future vie professionnelle sont tout de même considérées très pertinentes ou pertinentes par la plupart des répondants. C'est le cas, par exemple, de la réalisation d'une affiche que neuf étudiants trouvent très pertinente ou pertinente bien que cette activité se retrouve au sixième rang en terme d'importance. En ce qui concerne les technologies, seule l'utilisation de l'outil de gestion de notices bibliographiques est remise en cause, et ce, par seulement deux étudiants.

- Trois étudiants jugent que la critique constructive devrait être couverte dans un cours de communication et non dans un cours de spécialité. Le questionnaire ne permet cependant pas de vérifier si ces étudiants estiment que cette habileté est suffisamment importante pour être incluse dans un cours obligatoire et non dans un cours optionnel de spécialité ou s'ils s'objectent à un décloisonnement des spécialités (communication vs ingénierie).

\section{Retour sur le fondement socioconstructiviste de l'activité}

Tout au long de l'activité, les étudiants vivent des conflits cognitifs et même affectifs qui les conduisent à remettre en question leurs connaissances et leurs croyances. Par exemple, au début du cours, tous les étudiants croient que le prototypage rapide ne sert quà fabriquer des prototypes. Imaginez leur surprise lorsqu'ils critiquent un rapport où ils apprennent que certaines technologies permettent de fabriquer des moules en acier à partir desquels seront produites des pièces fonctionnelles en plastique! En cherchant, lisant et organisant l'information nécessaire à la rédaction de leur rapport, ils enrichissent leur conception de ce qu'est le prototypage rapide. Grâce à la critique constructive, ils s'aperçoivent que leurs pairs sont capables de jugement critique tout aussi valable et pertinent que celui de l'expert. À la lecture des critiques qui leur sont adressées, force est d'admettre que leur compétence de communication n'est pas nécessairement à un niveau satisfaisant. Ces exemples ne sont que quelques-uns parmi tant d'autres 
démontrant que les étudiants sont les acteurs de leur propre apprentissage et qu'il y a réellement construction, par opposition à transmission, de savoir.

Une telle activité perdrait son sens sans l'interaction avec les pairs, s'il n'y avait pas de volet social. Mais il y a plus. Une activité socioconstructiviste se doit également de stimuler des interactions avec le milieu. Au cours de leur vie professionnelle, les futurs ingénieurs seront appelés à travailler dans des équipes géographiquement distribuées où le partage d'information se fera électroniquement plutôt que sur papier. Ils doivent donc maîtriser toute une panoplie d'outils et de méthodes de travail leur permettant de rechercher, d'organiser, de produire et de diffuser de l'information sous forme électronique. La réalisation de l'activité de séminaire nécessite sept technologies différentes qui, sauf possiblement le logiciel EndNote, font partie de l'univers de travail d'un ingénieur.

\section{Conclusion}

L'organisation d'une activité nommée « séminaire» dans le cours «MEC626 - Prototypage rapide» a permis de faire vivre aux étudiants une situation d'apprentissage motivante d'inspiration socioconstructiviste. Grâce à cette activité, jumelée à l'utilisation judicieuse de la technologie, ils ont non seulement fait l'apprentissage de nouveaux outils, mais développé de nouvelles compétences méthodologiques. Les étudiants ont produit une documentation francophone à jour et de très grande qualité. Tout au long de l'activité, ils ont été confrontés à des situations où ils ont dû revoir leurs conceptions et ainsi construire leur savoir. Habitués à effectuer des recherches sur le Web, mais ignorants des ressources mises à leur disposition par la bibliothèque, les étudiants ont élargi leurs sources d'information. De plus, submergés par l'information, ils ont appris à raffiner leurs techniques de recherche, à juger de la qualité de l'information et à classifier celle-ci à l'aide d'un logiciel de gestion de notices bibliographiques. Ils ont développé leur sens critique tout en découvrant certaines fonctionnalités de Word leur permettant de critiquer un texte. Ils ont fait usage du transfert FTP pour échanger des fichiers. Ce mode d'échange d'information s'est révélé particulièrement efficace. Comparativement au courriel, le site FTP a servi de vitrine où les étudiants du cours ont pu récupérer et consulter les travaux de leurs collègues à loisir, rehaussant l'aspect social de l'activité. Par comparaison au dépôt des documents sur le site Web du cours, le transfert FTP a également libéré la professeure de la tâche de mise à jour du site. Finalement, les étudiants ont appris à produire une affiche électronique, ajoutant un nouveau mode de communication à leur arsenal.

Au total, sept technologies de l'information et de la communication ont été mises en œuvre pour soutenir l'activité, contribuant à sa pertinence, car ce sont des technologies couramment utilisées dans la pratique professionnelle. Ces technologies ont facilité l'échange d'information, ont amélioré la productivité du groupe ainsi que la qualité des productions. Il semble que les étudiants aient également ressenti les avantages de la technologie, car lors des différentes évaluations, celles-ci n'ont fait l'objet que d'un très faible nombre de critiques négatives.

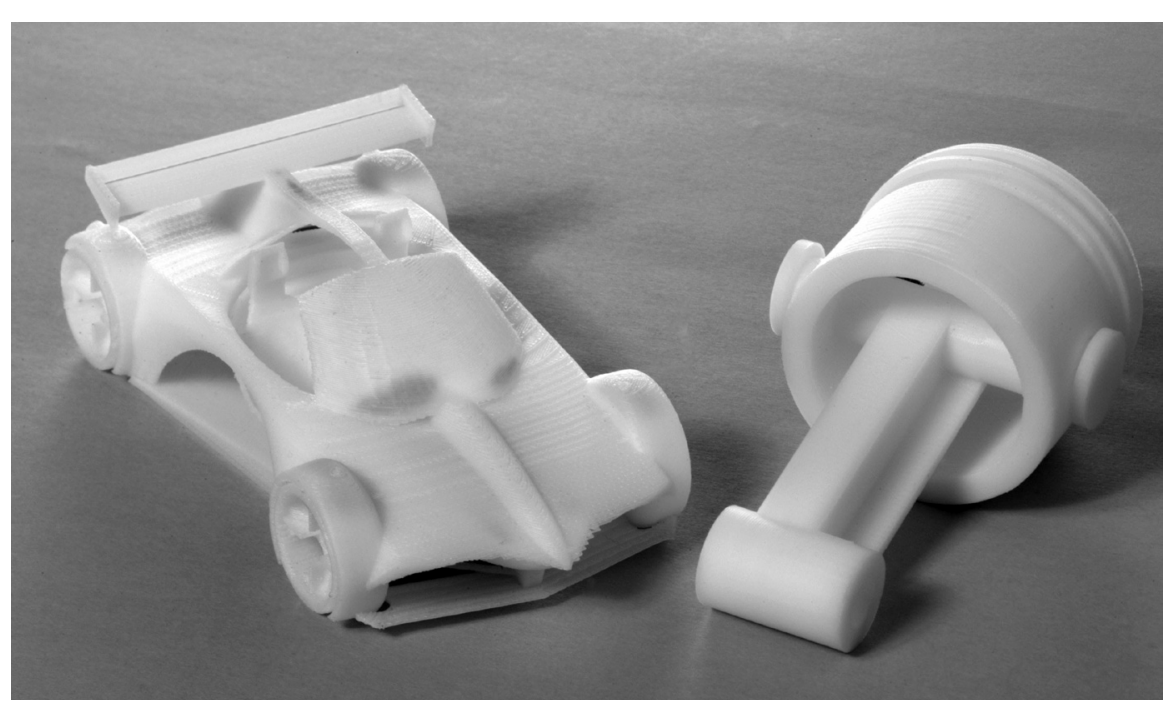

Figure 1. Exemple de pièces produites par les étudiants

\section{Annexe 1}

Dans le cas qui nous intéresse, le prototypage rapide fait référence à un ensemble de technologies de fabrication à l'aide desquelles un objet est fabriqué par ajout de matière, couche par couche. Parmi les procédés les plus utilisés en Amérique du Nord, on retrouve:

- le SLA (stéréolithographie), le MJM (multijet modeling ou fabrication multijets) et le SLS (selective laser sintering ou frittage sélectif au laser) de la compagnie 3D Systems (http://www.3dsystems.com/);

- le FDM (fused deposition modeling) de la compagnie Stratasys

(http://www.stratasys.com/);

- l'impression 3D (3D-Printing) de l'université MIT licencée à la compagnie Z Corp (http://www.zcorp.com/).

À l'été 2002, l'ÉTS a fait l'acquisition d'une machine Dimension de la compagnie Stratasys. Les étudiants l'utilisent pour fabriquer leurs propres prototypes, dont voici quelques exemples (voir la Figure 1).

L'expression «prototypage rapide» est aussi utilisée pour désigner une méthodologie, surtout employée en génie logiciel, servant à préciser les besoins des clients grâce à la manipulation de prototypes. Cet aspect n'est pas couvert dans le cours MEC626. 


\section{Références}

Conseil canadien des ingénieurs (2003). Des ingénieurs canadiens pour l'avenir - Inscriptions et diplômes décernés en génie - Tendances. Récupéré le 8 novembre 2004 de http://www.ccpe.ca/f/ files/report_enrolment_fr.pdf

Glasersfeld, E. (1994). Pourquoi le constructivisme doit-il être radical? Revue des sciences de l'éducation, 20(1), 21-28.

Lafortune, L. et Deaudelin, C. (2002). Accompagnement socioconstructiviste: pour s'approprier une réforme en éducation. Sainte-Foy: Presses de l'Université du Québec.

Mittermeyer, D. et Quirion, D. (2003). Étude sur les connaissances en recherche documentaire des étudiants entrant au ler cycle dans les universités québécoises. Montréal : Conférence des recteurs et des principaux des universités du Québec [CREPUQ].

Perkins, D. N. (1991). Technology meets constructivism: Do they make a marriage? Educational Technology, 31(5), 18-23.

Sauvé, P. (2000). Le socioconstructivisme et les grandes orientations de la réforme [version électronique]. Virage Express, 2(3), 1-4. Récupéré le 8 novembre 2004 de www.meq.gouv.qc.ca/ virage/journal_fr/Express_2__3.pdf

\section{Note}

1 La nouvelle version du cours a été conçue à l'aide de la méthode d'ingénierie de systèmes d'apprentissage (MISA), mise au point au Laboratoire d'informatique cognitive et environnements de formation (LICEF - http://www.licef.teluq. uquebec.ca/) de la Téluq (université à distance, constituante du réseau de l'Université du Québec). MISA est une méthode d'ingénierie pédagogique qui, dans sa version 2.0, est constituée de six phases réparties dans le temps (définition du projet, analyse/conception préliminaire, architecture, conception du matériel pédagogique, production/validation, diffusion) au cours desquelles trois modèles sont raffinés. Ces trois modèles sont: le modèle de connaissances, représentant le contenu; le modèle pédagogique, proposant les activités d'apprentissage et les scénarios de formation et d'encadrement correspondants; le modèle médiatique à l'aide duquel sont définis les médias et l'infrastructure de diffusion. Le cours est maintenant structuré autour de six activités :

- le démarrage

- le séminaire

- la fabrication

- le système expert

- l'étude de cas

- les bilans. 